\title{
Microsensors for In-Situ Chemical, Physical, and Radiological Characterization of Mixed Waste
}

\section{Research Objective}

A widespread need exists for portable, real-time, in-situ chemical, physical, and radiological sensors for the characterization and monitoring of mixed waste, ground water, contaminated soil and process streams. The applications range from monitoring plume containment and the results of remediation efforts to the determination of the location, chemical composition, and level of contaminants in soil or groundwater. The objective of this research program is to gain a better understanding of the molecular-level mechanism of adsorption-induced stress on microcantilever sensors. The ability to manipulate and control these stresses will lead to the development of highly selective and extremely sensitive sensors for EM specific applications in liquid environment in the presence of interferents. Cantilevers can be made specific for chemical species by attaching molecular recognition agents to one surface of a cantilever so that adsorption induced stress primarily occurs at that surface. Furthermore, it is possible to separate chemisorption and physisorption by simultaneous measurement of the changes in resonance frequency and static deflection of a cantilever. Based on these parameters, this program has the objective of developing and demonstrating corrosion resistant cantilevers with parts-per-trillion sensitivity for metal ions in solution such as $\mathrm{Hg}^{2+}, \mathrm{CrO}_{4}{ }^{2-}, \mathrm{Sr}^{2+}$, and $\mathrm{TcO}_{4}{ }^{-}$. Selectivity will be achieved by orthogonal arraying of modified cantilevers. A prototype sensor platform having a sensor cantilever and a reference cantilever will be field-tested in the third year, and this basic platform is intended to be the basis for a plethora of inexpensive, miniature sensors for a variety of heavy metal contaminants in solution.

\section{Research Progress and Implications}

This report summarizes the results in June 2002, in the second year of a three-year project. The three basic objectives of this program are to advance the fundamental understanding of the molecularly specific adsorption induced deflection of cantilevers, to conduct developmental research toward chemical species-specific sensors, and to field test a prototype microcantilever sensor in contaminated groundwater. Progress has been made along all three fronts since the beginning of this project. In the past year sensor elements have been demonstrated for $\mathrm{Hg}^{2+}$ and $\mathrm{Hg}\left(\mathrm{CH}_{3}\right)^{+}$in solution, potentially allowing dissolved $\mathrm{Hg}$ in solution to not only be quantified for total $\mathrm{Hg}$ but also for speciation. Based on this research, a new program to field test a $\mathrm{Hg}$ (II) sensor was established. Sensors that are specific for $\mathrm{Ca}^{2+}$ and $\mathrm{Cu}^{2+}$ in the presence of a variety of potential interferents were also demonstrated.

Fundamental Cantilever Research. Adsorption was observed to induce changes in the spring constant of a cantilever, leading to errors in the calculation of adsorbed mass from shifts in resonance frequency. Simultaneous measurement of resonance frequency and adsorption induced bending was shown to allow the change in spring constant to be determined. This change in spring constant occurs with high $\mathrm{NaCl}$ concentrations $(0.05$ to $0.8 \mathrm{M}$ ) in solution. The adsorption of $\mathrm{Ca}^{2+}$ on a silicon nitride cantilever was attributed to interaction of the positively charged ions with ionized siloxane functional groups on the cantilever surface. 
Metal Ion Specific Sensor Elements. A silicon microcantilever with gold coated on one side was found to respond selectively and sensitively to $\mathrm{Hg}$ (II) ions in solution.

Modification of the Si surface with a silane reagent did not change the response to $\mathrm{Hg}$ whereas modification of the Au surface with octanethiol greatly retarded the rate of deflection indicating the $\mathrm{Hg}$ (II) is reacting with the Au surface. The surface charge on the Au-solution interface is postulated to reduce $\mathrm{Hg}$ (II) to a surface amalgam.

Modification of the gold surface with a monolayer1,6-hexanedithiol makes the surface sensitive and selective for $\left(\mathrm{CH}_{3}\right) \mathrm{Hg}^{+}$adsorption induced deflection. $\mathrm{Na}^{+}, \mathrm{K}^{+}, \mathrm{Pb}^{2+}, \mathrm{Zn}^{2+}$, $\mathrm{Cd}^{2+}, \mathrm{Ca}^{2+}$, and $\mathrm{Ni}^{2+}$ in solution do not interfere with the response of the microcantilever to Hg. Gold coated cantilevers with chemically modified surfaces respond sensitively to $\mathrm{Ca}^{2+}$ ions at a concentration of $10^{-9} \mathrm{M}$. The sorption of a monolayer of 2-(4mercaptophenoxy)-N,N-diethyl-acetamide as well as the agent bis (11-mercaptoundecyl) phosphate were shown to detect $\mathrm{Ca}^{2+}$ ions although the former was more selective. A self-assembled monolayer of L-cysteine on a cantilever coated with gold on one side was shown to be effective for the detection of a concentration of $10^{-10} \mathrm{M} \mathrm{Cu}^{2+}$. Both the $\mathrm{Ca}^{2+}$ and the $\mathrm{Cu}^{2+}$ were relatively free from interference by each other and $\mathrm{Na}^{+}, \mathrm{K}^{+}, \mathrm{Pb}^{2+}, \mathrm{Zn}^{2+}$, $\mathrm{Cd}^{2+}$, and $\mathrm{Ni}^{2}$ in solution.

Field Demonstration of Microcantilever Sensor. Based on the Hg sensing results described above, a new project with the objective of demonstrating a $\mathrm{Hg}$ sensor for contaminated surface water at the DOE Y12 National Security Complex began this FY with the objective of successfully demonstrating the technology by the end of calendar year 2002. In conducting this field test, a prototype sensor platform having one cantilever as the sensor cantilever and second cantilever as a reference will be developed. This platform will be utilized for a field demonstration of a chemically modified microcantilever sensor.

\section{Planned Activities}

In the remainder of this $\mathrm{FY}$ efforts will be concentrated on the sensing of $\mathrm{ReO}_{4}{ }^{-}$and $\mathrm{ClO}_{4}{ }^{-}$ in solution as stand-ins for the radioactive $\mathrm{TcO}_{4}{ }^{-}$species. In $\mathrm{FY} 2003$ efforts will focus on the development of sensors for $\mathrm{Sr}^{2+}, \mathrm{Pb}^{2+}$, and on a means of electrochemically removing sorbed $\mathrm{Hg}$ from the gold coating on the microcantilever. The platform utilized for the Hg sensor at DOEs Y12 site will be utilized for the field measurement of another contaminant on the Oak Ridge Reservation.

\section{Information Access}

"Ultrasensitive Detection of $\mathrm{CrO}_{4}{ }^{2-}$ Using Microcantilever Sensors," H-F. Ji. T. Thundat, R. Dabestani, G. M. Brown, P. F. Britt, and P. V. Bonnesen, Anal. Chem. 73, 1572-1577 (2001).

"Detection of pH variation Using Modified Microcantilever Sensors" H.-F. Ji, K. M. Hansen, Z. Hu, and T. Thundat, Sensors and Actuators B72, 233-238 (2001).

"Analysis of Amplifications of Thermal Vibrations of a Microcantilever" G. Muralidharan, A. Mehta, S. Cherian, and T. Thundat, J. Appl. Phys. 89, 4587-91 (2001).

Determination of Adsorption-Induced variation in the Spring Constant of a Microcantilever," S. Cherian and T. Thundat, Appl. Phys. Lett., 80, 2219-2221 (2002). 
"In situ Detection of Calcium Ions with Chemically Modified Microcantilevers," H-F. Ji. and T. Thundat, Sensors and Actuators, in press.

"Investigating the Mechanical Effects of Adsorption of $\mathrm{Ca}^{2+}$ Ions on a Silicon Nitride Microcantilever Surface," S. Cherian, A. Mehta, and T. Thundat, Langmuir, in press.

"Ultrasensitive Detection of $\mathrm{Hg}^{2+}$ Using Microcantilever Sensors," Xiaohe $\mathrm{Xu}$, Thomas G. Thundat, Gilbert M. Brown and Hai-Feng Ji

"Ultrasensitive Detection of $\mathrm{Cu}^{2+}$ Using a Microcantilever Sensor Modified with LCysteine Self-Assembled Monolayer," Xiaohe Xu, Na Zhang, Thomas G. Thundat, Gilbert M. Brown, and Hai-Feng Ji, submitted for publication. 